\title{
On computing inner-outer factorizations of periodic systems
}

\author{
Andreas Varga
}

\begin{abstract}
A numerically reliable state space algorithm is proposed for computing inner-outer factorizations of causal periodic descriptor systems. The main computational ingredients are the computation of a special condensed Kronecker-like form of periodic pairs using orthogonal reduction algorithms and the solution of periodic Riccati equations. The proposed approach is completely general, being applicable to arbitrary causal periodic systems with time-varying state dimensions.
\end{abstract}

\section{INTRODUCTION}

The inner-outer factorization is a powerful tool which is useful in many applications in control theory of both standard and periodic systems, including the solution of spectral factorization widely used in optimal control, signal processing and communication, normalized coprime factorization with applications to $\nu$-gap norm computation, solution of loop transfer recovery problems and $H_{2} / H_{\infty}$-optimal model matching synthesis problems of controllers or fault detection filters.

The periodic inner-outer factorization (PIOF) of a causal periodic system can be defined as a series coupling of a causal periodic inner system and a causal periodic outer system, or equivalently, in terms of the product representation of the corresponding proper transfer function matrices of appropriate lifted representations of these systems (see next session). The existing computational algorithms employ either lifting-based approaches relying on reliable algorithms for standard discrete-time systems [1] or state-space methods developed for special cases of periodic systems, as for example, periodic systems with injective input-output maps and without zeros on the unit circle [2]. Besides excessive computational burden, the lifting based approaches are not preserving the structure of the lifted system representations and therefore needs to additionally determine periodic realizations of the outer and inner factors, which involve delicate numerical computations [3].

In this paper we propose a general algorithm to compute the PIOF of arbitrary causal periodic systems. The main novelty with respect to the approach described in [2] for standard periodic systems is its ability to compute the PIOF for causal periodic descriptor systems with arbitrary zeros and time-varying state dimensions. Also there are no restrictions regarding the injectivity of the system input-output map. The main computations consist in the reduction of the original problem to a simple case by employing a special deflating technique and the solution of the associated periodic Riccati

A. Varga is with German Aerospace Center, DLR-Oberpfaffenhofen, Institute of Robotics and Mechatronics, D-82234 Wessling, Germany, andras.vargaedlr.de equation of reduced dimension. The underlying computational techniques employ structure exploiting and structure preserving algorithms, which directly lead to state-space realizations of the factors. The employed computational algorithms are numerically reliable, by using extensively reduction techniques based on orthogonal transformations.

\section{PRELIMINARIES}

We consider linear periodic time-varying discrete-time descriptor systems of the form

$$
\begin{aligned}
E_{k} x(k+1) & =A_{k} x(k)+B_{k} u(k) \\
y(k) & =C_{k} x(k)+D_{k} u(k)
\end{aligned}
$$

where the matrices $E_{k} \in \mathbb{R}^{\nu_{k} \times n_{k+1}}, A_{k} \in \mathbb{R}^{\nu_{k} \times n_{k}}, B_{k} \in$ $\mathbb{R}^{\nu_{k} \times m}, C_{k} \in \mathbb{R}^{p \times n_{k}}, D_{k} \in \mathbb{R}^{p \times m}$ are periodic with period $N \geq 1$. The periodic system (1) will be alternatively denoted by the periodic quintuple $\mathcal{S}:=\left(E_{k}, A_{k}, B_{k}, C_{k}, D_{k}\right)$. We assume that $\sum_{k=1}^{N} \nu_{k}=\sum_{k=1}^{N} n_{k}$ is always fulfilled. Periodic systems of this general forms have been considered in several works (see for example [4], [5], [6], [7]). In what follows we summarize some notations and definitions for periodic systems used throughout the paper.

\section{A. Causal periodic systems}

The case of causal systems, when $\nu_{k}=n_{k+1}$ and $E_{k}$ are invertible matrices, plays an important role in most of applications. For a causal system (1), we denote the monodromy matrix at time $k$ by

$\Psi_{E_{k}^{-1} A_{k}}:=E_{k+N-1}^{-1} A_{k+N-1} E_{k+N-2}^{-1} A_{k+N-2} \cdots E_{k}^{-1} A_{k}$.

The eigenvalues of $\Psi_{E_{k}^{-1} A_{k}}$ are called characteristic multipliers of the periodic pair $\left(E_{k}, A_{k}\right)$. Let $\mathbb{D}$ denote the interior of the unit disk centered in the origin and let $\overline{\mathbb{D}}$ denote its closure including the points on the unit circle too. We denote $\mathbb{D}_{c}$ the complement of $\overline{\mathbb{D}}$ with respect to the complex plane $\mathbb{C}$ (i.e., $\mathbb{D}_{c}=\mathbb{C} \backslash \overline{\mathbb{D}}$ ). We say that $\mathcal{S}$ is stable (or equivalently the periodic pair $\left(E_{k}, A_{k}\right)$ is stable) if all characteristic multipliers have moduli less then one (i.e., $\left.\Lambda\left(\Psi_{E_{k}^{-1} A_{k}}\right) \subset \mathbb{D}\right)$. Note that $\Psi_{E_{k}^{-1} A_{k}}$ has always $n_{k}-\underline{n}$ null eigenvalues, where $\underline{n}:=\min \left\{n_{k}\right\}$. The rest of $\underline{n}$ eigenvalues of $\Psi_{E_{k}^{-1} A_{k}}$ form the core characteristic multipliers and are the same for all values of $k$. For non-causal systems (e.g., with $E_{k}$ singular or even non-square), similar notions like finite and infinite characteristic multipliers can be defined using lifting-based representations (see for example [5]).

The definitions used for reachability and observability of causal periodic descriptor systems are those of [4], which also apply for the more general non-causal case. For causal systems, we will use the following simpler definitions of 
stabilizability and detectability. The causal periodic system $\mathcal{S}$ is stabilizable if there exists a periodic $F_{k}$ of appropriate dimensions such that the periodic pair $\left(E_{k}, A_{k}+B_{k} F_{k}\right)$ is stable. Similarly, the causal periodic system $\mathcal{S}$ is detectable if there exists a periodic $L_{k}$ of appropriate dimensions such that the periodic pair $\left(E_{k}, A_{k}+L_{k} C_{k}\right)$ is stable.

\section{B. Similarity transformations}

Two periodic systems $\mathcal{S}:=\left(E_{k}, A_{k}, B_{k}, C_{k}, D_{k}\right)$ and $\widetilde{\mathcal{S}}:=\left(\widetilde{E}_{k}, \widetilde{A}_{k}, \widetilde{B}_{k}, \widetilde{C}_{k}, D_{k}\right)$ are called similar if the matrices of their state-space representations are related by

$$
\widetilde{E}_{k}=U_{k} E_{k} V_{k+1}, \widetilde{A}_{k}=U_{k} A_{k} V_{k}, \widetilde{B}_{k}=U_{k} B, \widetilde{C}_{k}=C_{k} V_{k},
$$

with $U_{k}$ and $V_{k} N$-periodic nonsingular matrices. Two similar systems have the same input-output map.

\section{System couplings}

The series coupling of two periodic systems $\mathcal{S}_{1}=\left(E_{k}^{(1)}, A_{k}^{(1)}, B_{k}^{(1)}, C_{k}^{(1)}, D_{k}^{(1)}\right) \quad$ and $\quad \mathcal{S}_{2}=$ $\left(E_{k}^{(2)}, A_{k}^{(2)}, B_{k}^{(2)}, C_{k}^{(2)}, D_{k}^{(2)}\right)$ we denote with $\mathcal{S}_{1} \star \mathcal{S}_{2}:=$ $\left(\widehat{E}_{k}, \widehat{A}_{k}, \widehat{B}_{k}, \widehat{C}_{k}, \widehat{D}_{k}\right)$ and has a realization with the state-space matrices

$$
\begin{gathered}
\widehat{E}_{k}=\left[\begin{array}{cc}
E_{k}^{(1)} & 0 \\
0 & E_{k}^{(2)}
\end{array}\right], \quad \widehat{A}_{k}=\left[\begin{array}{cc}
A_{k}^{(1)} & B_{k}^{(1)} C_{k}^{(2)} \\
0 & A_{k}^{(2)}
\end{array}\right], \\
\widehat{B}_{k}=\left[\begin{array}{c}
B_{k}^{(1)} D_{k}^{(2)} \\
B_{k}^{(2)}
\end{array}\right], \quad \widehat{C}_{k}=\left[\begin{array}{ll}
C_{k}^{(1)} & D_{k}^{(1)} C_{k}^{(2)}
\end{array}\right], \\
\widehat{D}_{k}=D_{k}^{(1)} D_{k}^{(2)}
\end{gathered}
$$

A similar realization corresponds to the parallel coupling of two periodic systems $\mathcal{S}_{1}$ and $\mathcal{S}_{2}$ denoted by $\mathcal{S}_{1} \oplus \mathcal{S}_{2}$.

We denote by $\left[\mathcal{S}_{1} \mathcal{S}_{2}\right]$ and $\left[\begin{array}{l}\mathcal{S}_{1} \\ \mathcal{S}_{2}\end{array}\right]$ the input and output concatenated couplings, respectively, which have obvious state-space representations. For example, the realization of $\left[\mathcal{S}_{1} \mathcal{S}_{2}\right]:=\left(\widehat{E}_{k}, \widehat{A}_{k}, \widehat{B}_{k}, \widehat{C}_{k}, \widehat{D}_{k}\right)$ has the matrices

$$
\begin{gathered}
\widehat{E}_{k}=\left[\begin{array}{cc}
E_{k}^{(1)} & 0 \\
0 & E_{k}^{(2)}
\end{array}\right], \quad \widehat{A}_{k}=\left[\begin{array}{cc}
A_{k}^{(1)} & 0 \\
0 & A_{k}^{(2)}
\end{array}\right], \\
\widehat{B}_{k}=\left[\begin{array}{cc}
B_{k}^{(1)} & 0 \\
0 & B_{k}^{(2)}
\end{array}\right], \quad \widehat{C}_{k}=\left[\begin{array}{cc}
C_{k}^{(1)} & C_{k}^{(2)}
\end{array}\right], \\
\widehat{D}_{k}=\left[\begin{array}{ll}
D_{k}^{(1)} & D_{k}^{(2)}
\end{array}\right]
\end{gathered}
$$

\section{Inversion and conjugation}

The inverse of $\mathcal{S}=\left(E_{k}, A_{k}, B_{k}, C_{k}, D_{k}\right)$ for invertible $D_{k}$ is

$$
\mathcal{S}^{-1}=\left(E_{k}, A_{k}-B_{k} D_{k}^{-1} C_{k}, B_{k} D_{k}^{-1},-D_{k}^{-1} C_{k}, D_{k}^{-1}\right)
$$

More general inverses of periodic systems are discussed in [7].

The conjugate system $\mathcal{S}^{\sim}$ is defined as $\mathcal{S}^{\sim}=$ $\left(\widetilde{E}_{k}, \widetilde{A}_{k}, \widetilde{B}_{k}, \widetilde{C}_{k}, \widetilde{D}_{k}\right)$, where (see [8])

$$
\widetilde{E}_{k}=\left[\begin{array}{cc}
A_{k}^{T} & 0 \\
-B_{k}^{T} & 0
\end{array}\right], \quad \widetilde{A}_{k}=\left[\begin{array}{cc}
E_{k-1}^{T} & 0 \\
0 & -I
\end{array}\right],
$$

$$
\widetilde{B}_{k}=\left[\begin{array}{c}
-C_{k}^{T} \\
D_{k}^{T}
\end{array}\right], \quad \widetilde{C}_{k}=\left[\begin{array}{ll}
0 & I
\end{array}\right], \quad \widetilde{D}_{k}=0
$$

\section{E. Lifted representation}

We can formulate the factorization problems addressed in this paper and interpret the obtained results in terms of the transfer-function matrix (TFM) corresponding to the associated stacked lifted representation of [9], which uses the input-state-output behavior of the system over time intervals of length $N$, rather then 1 . The lifted input, output and state vectors are defined as

$$
\begin{aligned}
& \bar{u}_{k}(h)=\left[u^{T}(h N+k) \cdots u^{T}(h N+k+N-1)\right]^{T}, \\
& \bar{y}_{k}(h)=\left[y^{T}(h N+k) \cdots y^{T}(h N+k+N-1)\right]^{T}, \\
& \bar{x}_{k}(h)=\left[x^{T}(h N+k) \cdots x^{T}(h N+k+N-1)\right]^{T}
\end{aligned}
$$

and the corresponding lifted system at time moment $k$ can be represented by a linear time-invariant (LTI) discrete-time descriptor system of the form

$$
\begin{aligned}
\bar{E}_{k}^{\mathcal{S}} \bar{x}_{k}(h+1) & =\bar{A}_{k}^{\mathcal{S}} \bar{x}_{k}(h)+\bar{B}_{k}^{\mathcal{S}} \bar{u}_{k}(h) \\
\bar{y}_{k}(h) & =\bar{C}_{k}^{\mathcal{S}} \bar{x}_{k}(h)+\bar{D}_{k}^{\mathcal{S}} \bar{u}_{k}(h)
\end{aligned}
$$

where

$$
\bar{A}_{k}^{\mathcal{S}}-z \bar{E}_{k}^{\mathcal{S}}=\left[\begin{array}{ccccc}
A_{k} & -E_{k} & O & \cdots & O \\
O & \ddots & \ddots & \ddots & \vdots \\
\vdots & \ddots & \ddots & -E_{k+N-3} & O \\
O & & \ddots & A_{k+N-2} & -E_{k+N-2} \\
-z E_{k+N-1} & O & \cdots & O & A_{k+N-1}
\end{array}\right]
$$

is the pole pencil of the periodic pair $\left(A_{k}, E_{k}\right)$ and

$$
\begin{aligned}
& \bar{B}_{k}^{\mathcal{S}}=\operatorname{diag}\left\{B_{k}, \ldots, B_{k+N-1}\right\}, \\
& \bar{C}_{k}^{\mathcal{S}}=\operatorname{diag}\left\{C_{k}, \ldots, C_{k+N-1}\right\}, \\
& \bar{D}_{k}^{\mathcal{S}}=\operatorname{diag}\left\{D_{k}, \ldots, D_{k+N-1}\right\} .
\end{aligned}
$$

The $p N \times m N$ TFM of the lifted-system at time moment $k$ corresponding to $\mathcal{S}$ we denote by

$$
G_{k}^{\mathcal{S}}(z)=\bar{C}_{k}^{\mathcal{S}}\left(z \bar{E}_{k}^{\mathcal{S}}-\bar{A}_{k}^{\mathcal{S}}\right)^{-1} \bar{B}_{k}^{\mathcal{S}}+\bar{D}_{k}^{\mathcal{S}}
$$

It can be shown that the TFM $G_{k}^{\mathcal{S}}(z)$ of a causal system (i.e., $E_{k}$ invertible) belongs to a special type of proper TFMs for which $G_{k}^{\mathcal{S}}(\infty)$ is finite and has a lower block-triangular structure, with all diagonal blocks of the form $p \times m$. Only proper TFMs with this special property correspond to causal periodic systems.

The previously defined operations with periodic systems: series coupling, parallel coupling, inversion (2), or conjugation, can easily be expressed in terms of TFMs as follows:

$$
\begin{gathered}
G_{k}^{\mathcal{S}_{1} \star \mathcal{S}_{2}}(z)=G_{k}^{\mathcal{S}_{1}}(z) G_{k}^{\mathcal{S}_{2}}(z), \\
G_{k}^{\mathcal{S}_{1} \oplus \mathcal{S}_{2}}(z)=G_{k}^{\mathcal{S}_{1}}(z)+G_{k}^{\mathcal{S}_{2}}(z), \\
G_{k}^{\mathcal{S}^{-1}}(z)=\left(G_{k}^{\mathcal{S}}(z)\right)^{-1}, \\
G_{k}^{\mathcal{S}^{\sim}}(z)=\left(G_{k}^{\mathcal{S}}(1 / z)\right)^{T} .
\end{gathered}
$$

In what follows we assume that the lifting of input and output concatenated systems is done by preserving the original 
separation of the inputs and outputs (instead of lifting jointly the inputs and outputs). This has the important consequence that the lifted TFM of an input concatenated system $\left[\mathcal{S}_{1} \mathcal{S}_{2}\right]$ can be built by concatenating the individual lifted TFMs column-wise, i.e.

$$
G_{k}^{\left[\mathcal{S}_{1} \mathcal{S}_{2}\right]}(z)=\left[\begin{array}{ll}
G_{k}^{\mathcal{S}_{1}}(z) & G_{k}^{\mathcal{S}_{2}}(z)
\end{array}\right]
$$

Similarly, the lifted TFM of output concatenated systems can be built by stacking the individual lifted TFMs row-wise.

\section{F. System zeros, left/right Kronecker structures}

The zeros of the periodic system $\mathcal{S}$ can be defined as the zeros of the lifted TFM $G_{k}^{\mathcal{S}}(z)$ and thus of the associated system pencil

$$
P_{k}^{\mathcal{S}}(z)=\left[\begin{array}{cc}
\bar{A}_{k}^{\mathcal{S}}-z \bar{E}_{k}^{\mathcal{S}} & \bar{B}_{k}^{\mathcal{S}} \\
\bar{C}_{k}^{\mathcal{S}} & \bar{D}_{k}^{\mathcal{S}}
\end{array}\right]
$$

We call the periodic system $\mathcal{S}$ minimal phase if $P_{k}^{\mathcal{S}}(z)$ has only zeros in $\mathbb{D}$. This pencil also provide information on the left and right Kronecker structure of $G_{k}^{\mathcal{S}}(z)$. For example, to a full column rank $G_{k}^{\mathcal{S}}(z)$ corresponds a system pencil $P_{k}^{\mathcal{S}}(z)$ without right Kronecker structure. For further details on the determination of system zeros and the Kronecker structure see Appendix A.

\section{THE PERIODIC INNER-OUTER FACTORIZATION}

In this paper we consider the computation of a factorized representation of a causal periodic system $\mathcal{S}$ in the form

$$
\begin{aligned}
\mathcal{S} & =\mathcal{S}_{i} \star \mathcal{S}_{o} \\
& :=\left[\mathcal{S}_{i, 1} \mathcal{S}_{i, 2}\right] \star\left[\begin{array}{c}
\mathcal{S}_{o, 1} \\
0
\end{array}\right] \\
& =\mathcal{S}_{i, 1} \star \mathcal{S}_{o, 1}
\end{aligned}
$$

where $\mathcal{S}_{i}$ and $\mathcal{S}_{o, 1}$ are periodic systems with special properties. The lifted TFMs corresponding to the factorized representation (6) can be expressed similarly as

$$
\begin{aligned}
G_{k}^{\mathcal{S}}(z) & =G_{k}^{\mathcal{S}_{i}}(z) G_{k}^{\mathcal{S}_{o}}(z) \\
& =\left[G_{k}^{\mathcal{S}_{i, 1}}(z) G_{k}^{\mathcal{S}_{i, 2}}(z)\right]\left[\begin{array}{c}
G_{k}^{\mathcal{S}_{o, 1}}(z) \\
0
\end{array}\right] \\
& =G_{k}^{\mathcal{S}_{i, 1}}(z) G_{k}^{\mathcal{S}_{o, 1}}(z)
\end{aligned}
$$

The factorization $\mathcal{S}=\mathcal{S}_{i, 1} \star \mathcal{S}_{o, 1}$ in (6) is called an periodic inner-outer factorization (PIOF) if $G_{k}^{\mathcal{S}_{i, 1}}(z)$ is an inner TFM satisfying $G_{k}^{\mathcal{S}_{i, 1}^{\sim}}(z) G_{k}^{\mathcal{S}_{i, 1}}(z)=I$, and $G_{k}^{\mathcal{S}_{o, 1}}(z)$ is an outer TFM, having full row rank and only zeros in the closed unit disk $\overline{\mathbb{D}}$. The full factorization $\mathcal{S}=\mathcal{S}_{i} \star \mathcal{S}_{o}$ with $G_{k}^{\mathcal{S}_{i}}(z)$ an inner and square TFM is useful in solving optimal controller and filter synthesis problems. The inner system $\mathcal{S}_{i, 2}$ is called the complementary inner factor and its conjugate $\mathcal{S}_{i, 2}^{\sim}$ is an all-pass left annihilator of the periodic system $\mathcal{S}$.

In the rest of the paper, we describe computational algorithms for the PIOF, where we address first the simple case of causal periodic systems with time-varying state dimensions without zeros on the unit circle and having injective inputoutput maps. Then, we discuss a general PIOF algorithm, whose main novelty is a preprocessing procedure by which the PIOF for an arbitrary causal periodic system is computed by determining the PIOF of a reduced order system using the simple case solution method. Both algorithms are numerically reliable and exclusively rely on structure preserving lifting-free computations.

\section{Computation of PIOF: The Simple CASE}

Let $\mathcal{S}=\left(E_{k}, A_{k}, B_{k}, C_{k}, D_{k}\right)$ be a causal periodic system with $E_{k}$ square and invertible. We assume that the realization of $\mathcal{S}$ is stabilizable and detectable. Further, we assume the lifted TFM $G_{k}^{\mathcal{S}}(z)$ has no zeros on the unit circle and has full column rank. The following result extends those of [2] to causal periodic descriptor systems with time-varying state dimensions.

Proposition 1: For the stabilizable and detectable system $\mathcal{S}=\left(E_{k}, A_{k}, B_{k}, C_{k}, D_{k}\right)$, let $X_{k}$ be the symmetric periodic stabilizing solution of the generalized periodic discrete-time algebraic Riccati equation (GPDARE)

$$
\begin{aligned}
& E_{k-1}^{T} X_{k} E_{k-1}=A_{k}^{T} X_{k+1} A_{k}+C_{k}^{T} C_{k} \\
& \quad-\left(A_{k}^{T} X_{k+1} B_{k}+C_{k}^{T} D_{k}\right) R_{k}^{-1}\left(B_{k}^{T} X_{k+1} A_{k}+D_{k}^{T} C_{k}\right)
\end{aligned}
$$

where $R_{k}=D_{k}^{T} D_{k}+B_{k}^{T} X_{k+1} B_{k}$, and let $F_{k}$ be the corresponding stabilizing periodic feedback matrix

$$
F_{k}=-R_{k}^{-1}\left(B_{k}^{T} X_{k+1} A_{k}+D_{k}^{T} C_{k}\right) .
$$

Then, the outer factor $\mathcal{S}_{o, 1}$ has the periodic state space realization

$$
\mathcal{S}_{o, 1}=\left(E_{k}, A_{k}, B_{k},-R_{k}^{\frac{1}{2}} F_{k}, R_{k}^{\frac{1}{2}}\right)
$$

and the inner factor $\mathcal{S}_{i, 1}$ can be expressed as

$$
\mathcal{S}_{i, 1}=\left(E_{k}, A_{k}+B_{k} F_{k}, B_{k} R_{k}^{-\frac{1}{2}}, C_{k}+D_{k} F_{k}, D_{k} R_{k}^{-\frac{1}{2}}\right)
$$

Proof. The assumption on stabilizability and detectability guarantees the existence of a unique stabilizing non-negative definite symmetric periodic solution $X_{k}$ of the GPDARE (7).

It is easy to show that $\mathcal{S}$ and $\mathcal{S}_{i, 1} \star \mathcal{S}_{o, 1}$ have the same input-output maps. Further, we can show that $\mathcal{S}_{o, 1}$ has only stable zeros, by explicitly forming the inverse system

$$
\mathcal{S}_{o, 1}^{-1}=\left(E_{k}, A_{k}+B_{k} F_{k}, B_{k} R_{k}^{-\frac{1}{2}}, F_{k}, R_{k}^{-\frac{1}{2}}\right)
$$

and observing that this periodic system has only stable characteristic multipliers, because the feedback $F_{k}$ in (8) is stabilizing.

To show that $\mathcal{S}_{i, 1}$ is inner, we use the extension of the characterization of inner factors for standard periodic systems (i.e., with $E_{k}=I$ ) in [10]. For this we check by direct computation that for the periodic realization of $\mathcal{S}_{i, 1}=\left(E_{k, i}, A_{k, i}, B_{k, i}, C_{k, i}, D_{k, i}\right)$ the following conditions are fulfilled:

$$
\begin{aligned}
A_{k, i}^{T} X_{k+1} A_{k, i}+C_{k, i}^{T} C_{k, i} & =E_{k-1, i}^{T} X_{k} E_{k-1, i} \\
B_{k, i}^{T} X_{k+1} A_{k, i}+D_{k, i}^{T} C_{k, i} & =0 \\
D_{k, i}^{T} D_{k, i}+B_{k, i}^{T} X_{k+1} B_{k, i} & =I
\end{aligned}
$$

For the numerical solution of the GPDARE, numerically reliable computational approaches are described in [11]. 
Remark. It is easy to show that $\mathcal{S}^{\sim} \star \mathcal{S}$ and $\mathcal{S}_{o, 1}^{\sim} \star \mathcal{S}_{o, 1}$ have the same input-output map. Therefore, if $\mathcal{S}$ is stable, then $\mathcal{S}_{o, 1}$ is the minimal phase stable spectral factor of $\mathcal{S}$. If $\mathcal{S}$ is not stable, then $\mathcal{S}_{o, 1}$ is the minimal phase factor of $\mathcal{S}$.

The complementary inner factor can be determined in a similar way as in the LTI case [12], by writing down the conditions (9) for the system $\left[\mathcal{S}_{i, 1} \mathcal{S}_{i, 2}\right]$, with a realization of $\mathcal{S}_{i, 2}$ chosen in the form

$$
\mathcal{S}_{i, 2}=\left(E_{k}, A_{k}+B_{k} F_{k}, Y_{k}, C_{k}+D_{k} F_{k}, Z_{k}\right)
$$

It follows that the periodic matrices $Y_{k}$ and $Z_{k}$ must satisfy the conditions

$$
\begin{aligned}
A_{k}^{T} X_{k+1} Y_{k}+C_{k}^{T} Z_{k} & =0 \\
B_{k}^{T} X_{k+1} Y_{k}+D_{k}^{T} Z_{k} & =0 \\
Z_{k}^{T} Z_{k}+Y_{k}^{T} X_{k+1} Y_{k} & =I
\end{aligned}
$$

Note that $Y_{k}$ and $Z_{k}$ are related to $F_{k}$ only implicitly through $X_{k}$.

The computation of $Y_{k}$ and $Z_{k}$ can be done as follows. First, determine for each $k$ an orthonormal basis $\left[\begin{array}{c}\widetilde{Y}_{k} \\ \widetilde{Z}_{k}\end{array}\right]$ of the right nullspace of the matrix $\left[\begin{array}{cc}A_{k}^{T} X_{k+1} & C_{k}^{T} \\ B_{k}^{T} & D_{k}^{T}\end{array}\right]$. Then, perform the normalization

$$
\left[\begin{array}{c}
Y_{k} \\
Z_{k}
\end{array}\right]=\left[\begin{array}{c}
\widetilde{Y}_{k} \\
\widetilde{Z}_{k}
\end{array}\right] H_{k}^{-1}
$$

where $H_{k}^{T} H_{k}=\widetilde{Z}_{k}^{T} \widetilde{Z}_{k}+\widetilde{Y}_{k}^{T} X_{k+1} \widetilde{Y}_{k}$.

\section{Computaton of PIOF: The general Case}

Consider a causal periodic system $\mathcal{S}=$ $\left(E_{k}, A_{k}, B_{k}, C_{k}, D_{k}\right)$ for which we only assume that is stabilizable. Let $Q_{k}$ and $Z_{k}$ be orthogonal $N$-periodic matrices determined using the procedure presented in Appendix B to reduce the $N$-periodic pair $\left(S_{k}, T_{k}\right)$ with

$$
S_{k}:=\left[\begin{array}{cc}
A_{k} & B_{k} \\
C_{k} & D_{k}
\end{array}\right], \quad T_{k}:=\left[\begin{array}{cc}
E_{k} & O \\
O & O
\end{array}\right]
$$

to the form $\left(\bar{S}_{k}, \bar{T}_{k}\right):=\left(Q_{k} S_{k} Z_{k}, Q_{k} T_{k} Z_{k+1}\right)$, where

$$
\bar{S}_{k}=\left[\begin{array}{ccc}
A_{k, r g} & * & * \\
0 & A_{k, b \ell} & B_{k, b \ell} \\
0 & C_{k, b \ell} & D_{k, b \ell}
\end{array}\right], \bar{T}_{k}=\left[\begin{array}{ccc}
E_{k, r g} & * & * \\
0 & E_{k, b \ell} & 0 \\
0 & 0 & 0
\end{array}\right]
$$

Let $G_{k}^{\mathcal{S}_{b \ell}}(z)$ be the lifted TFM of the periodic system

$$
\mathcal{S}_{b \ell}:=\left(E_{k, b \ell}, A_{k, b \ell}, B_{k, b \ell}, C_{k, b \ell}, D_{k, b \ell}\right)
$$

Since $G_{k}^{\mathcal{S}_{b \ell}}(z)$ has only zeros in $\mathbb{D}_{c}$ and has full column rank, we can apply the method for the simple case considered previously to compute the inner factor. The following result shows that the inner factor corresponding to $\mathcal{S}_{b \ell}$ is also the inner factor we seek for the original system, while the outer factor automatically results.

Theorem 1: For the stabilizable and detectable system $\mathcal{S}=\left(E_{k}, A_{k}, B_{k}, C_{k}, D_{k}\right)$, let $\mathcal{S}_{b \ell}$ defined in (11) be the reduced stabilizable system whose matrices are constructed in Appendix B. With obvious index replacements, let $X_{k, b \ell}$ be the periodic stabilizing solution of the associated periodic Riccati equation (7) and let $F_{k, b \ell}$ be the corresponding stabilizing periodic feedback. Then, the inner factor $\mathcal{S}_{i, 1}$ of the PIOF of $\mathcal{S}$ in (6) has the periodic realization

$$
\mathcal{S}_{i, 1}=\left(E_{k, b \ell}, \widetilde{A}_{k, b \ell}, \widetilde{B}_{k, b \ell}, \widetilde{C}_{k, b \ell}, \widetilde{D}_{k, b \ell}\right)
$$

with

$$
\begin{aligned}
\widetilde{A}_{k, b \ell} & =A_{k, b \ell}+B_{k, b \ell} F_{k, b \ell} \\
\widetilde{B}_{k, b \ell} & =B_{k, b \ell} R_{k, b \ell}^{-\frac{1}{2}} \\
\widetilde{C}_{k, b \ell} & =C_{k, b \ell}+D_{k, b \ell} F_{k, b \ell} \\
\widetilde{D}_{k, b \ell} & =D_{k, b \ell} R_{k, b \ell}^{-\frac{1}{2}}
\end{aligned}
$$

and the outer factor has the realization

$$
\mathcal{S}_{o, 1}=\left(E_{k}, A_{k}, B_{k}, \widetilde{C}_{k}, \widetilde{D}_{k}\right)
$$

where, with $Z_{k}$ defined in (15)

$$
\left[\begin{array}{ll}
\widetilde{C}_{k} & \widetilde{D}_{k}
\end{array}\right]=R_{k, b \ell}^{\frac{1}{2}}\left[\begin{array}{lll}
0 & -F_{k, b \ell} & I
\end{array}\right] Z_{k}^{T}
$$

Proof. We show successively that:

1) $\mathcal{S}$ and $\mathcal{S}_{i, 1} \star \mathcal{S}_{o, 1}$ are the same periodic system,

2) $\mathcal{S}_{o, 1}$ has only zeros in $\overline{\mathbb{D}}$,

3) $\mathcal{S}_{i, 1}$ is inner.

1) To prove that $\mathcal{S}=\mathcal{S}_{i, 1} \star \mathcal{S}_{o, 1}$, we use the realizations of $\mathcal{S}_{i, 1}$ and $\mathcal{S}_{o, 1}$ in (12) and (13), respectively, to build the realization of $\mathcal{S}_{i, 1} \star \mathcal{S}_{o, 1}$ defined by the periodic quintuple $\left(\widehat{E}_{k}, \widehat{A}_{k}, \widehat{B}_{k}, \widehat{C}_{k}, \widehat{D}_{k}\right)$, where

$$
\begin{aligned}
\widehat{E}_{k} & =\left[\begin{array}{cc}
E_{k, b \ell} & 0 \\
0 & E_{k}
\end{array}\right], \\
\widehat{A}_{k} & =\left[\begin{array}{cc}
A_{k, b \ell}+B_{k, b \ell} F_{k, b \ell} & B_{k, b \ell} \bar{C}_{k} \\
0 & A_{k}
\end{array}\right], \widehat{B}_{k}=\left[\begin{array}{c}
B_{k, b \ell} \bar{D}_{k} \\
B_{k}
\end{array}\right], \\
\widehat{C}_{k} & =\left[\begin{array}{cc}
C_{k, b \ell}+D_{k, b \ell} F_{k, b \ell} & D_{k, b \ell} \bar{C}_{k}
\end{array}\right], \widehat{D}_{k}=D_{k, b \ell} \bar{D}_{k}
\end{aligned}
$$

where

$$
\left[\begin{array}{ll}
\bar{C}_{k} & \bar{D}_{k}
\end{array}\right]=R_{k, b l}^{-\frac{1}{2}}\left[\begin{array}{ll}
\widetilde{C}_{k} & \widetilde{D}_{k}
\end{array}\right]=\left[\begin{array}{lll}
0 & F_{k, b \ell} & I
\end{array}\right] Z_{k}^{T}
$$

We perform on $\mathcal{S}_{i, 1} \star \mathcal{S}_{o, 1}$ a similarity transformation with periodic matrices of the form

$$
V_{k}=\left[\begin{array}{cc}
I & -Y_{k, 1} \\
0 & I
\end{array}\right], \quad W_{k}=\left[\begin{array}{cc}
I & Y_{k, 2} \\
0 & I
\end{array}\right]
$$

to obtain

$$
\begin{gathered}
V_{k} \widehat{E}_{k} W_{k+1}=\widehat{E}_{k}, \quad V_{k} \widehat{B}_{k}=\left[\begin{array}{c}
\underline{0} \\
B_{k}
\end{array}\right], \\
V_{k} \widehat{A}_{k} W_{k}=\left[\begin{array}{cc}
A_{k, b \ell}+B_{k, b \ell} F_{k, b \ell} & \underline{0} \\
0 & A_{k}
\end{array}\right], \\
\widehat{C}_{k} W_{k}=\left[\begin{array}{cc}
C_{k, b \ell}+D_{k, b \ell} F_{k, b \ell} & C_{k, e}
\end{array}\right],
\end{gathered}
$$

with $C_{k, e}=D_{k, b \ell} \bar{C}_{k}+\left(C_{k, b \ell}+D_{k, b \ell} F_{k, b \ell}\right) Y_{k, 2}$. The newly created zero blocks (underlined) show that $A_{k, b \ell}+$ $B_{k, b \ell} F_{k, b \ell}$ is non-reachable and the realization of $\mathcal{S}_{i, 1} \star \mathcal{S}_{o, 1}$ 
is $\left(E_{k}, A_{k}, B_{k}, C_{k, e}, D_{k, b \ell} \bar{D}_{k}\right)$. To achieve this, the periodic matrices $Y_{k, 1}$ and $Y_{k, 2}$ must satisfy the two matrix equations

$$
\begin{aligned}
{\left[\begin{array}{ll}
Y_{k, 1} & -B_{k, b \ell}
\end{array}\right]\left[\begin{array}{ll}
A_{k} & B_{k} \\
\bar{C}_{k} & \bar{D}_{k}
\end{array}\right] } & =\left(A_{k, b \ell}+B_{k, b \ell} F_{k, b \ell}\right)\left[\begin{array}{ll}
Y_{k, 2} & 0
\end{array}\right] \\
Y_{k, 1} E_{k} & =E_{k, b \ell} Y_{k+1,2}
\end{aligned}
$$

Since $E_{k, b \ell}$ is invertible, we can express

$$
Y_{k+1,2}=E_{k, b \ell}^{-1} Y_{k, 1} E_{k}
$$

By choosing $Y_{k, 1}=\left[\begin{array}{ll}0 & I\end{array}\right] U_{k}$, it is easy to see that

$$
\left[\begin{array}{ll}
Y_{k+1,2} & 0
\end{array}\right]=\left[\begin{array}{lll}
0 & I & 0
\end{array}\right] Z_{k+1}^{T}
$$

and also the first matrix equation is fulfilled. Also, $\left[\begin{array}{ll}C_{k, e} & D_{k, b \ell} \bar{D}_{k}\end{array}\right]$ can be expressed as

$$
\begin{aligned}
& D_{k, b \ell}\left[\bar{C}_{k} \bar{D}_{k}\right]+\left(C_{k, b \ell}+D_{k, b \ell} F_{k, b \ell}\right)\left[\begin{array}{ll}
Y_{k, 2} & 0
\end{array}\right]= \\
& {\left[\begin{array}{lll}
0 & C_{k, b \ell} & D_{k, b \ell}
\end{array}\right] Z_{k}^{T}=} \\
& {\left[\begin{array}{ll}
C_{k} & D_{k}
\end{array}\right]}
\end{aligned}
$$

2) To show that $\mathcal{S}_{o, 1}$ has only zeros in $\overline{\mathbb{D}}$ we show that the corresponding periodic pair $\left(\widetilde{S}_{k}, \widetilde{T}_{k}\right)$ defined as

$$
\widetilde{S}_{k}=\left[\begin{array}{cc}
A_{k} & B_{k} \\
\widetilde{C}_{k} & \widetilde{D}_{k}
\end{array}\right], \quad \widetilde{T}_{k}=\left[\begin{array}{cc}
E_{k} & 0 \\
0 & 0
\end{array}\right]
$$

has no zero structure corresponding to $\mathbb{D}_{c}$. This follows by computing

$$
\begin{gathered}
{\left[\begin{array}{cc}
U_{k} & 0 \\
0 & R_{k, b \ell}^{-\frac{1}{2}}
\end{array}\right] S_{k} Z_{k}=\left[\begin{array}{ccc}
A_{k, r g} & * & * \\
0 & A_{k, b \ell} & B_{k, b \ell} \\
0 & -F_{k, b \ell} & I
\end{array}\right]} \\
{\left[\begin{array}{cc}
U_{k} & 0 \\
0 & R_{k, b \ell}^{-\frac{1}{2}}
\end{array}\right] T_{k} Z_{k+1}=\left[\begin{array}{ccc}
E_{k, r g} & * & * \\
0 & E_{k, b \ell} & 0 \\
0 & 0 & 0
\end{array}\right]}
\end{gathered}
$$

The system zeros are partly those contained in the periodic pair $\left(A_{k, r g}, E_{k, r g}\right)$, which, by construction of the Kronecker-like form, all belong to $\overline{\mathbb{D}}$. The rest of the zeros corresponds to the invertible periodic system $\left(E_{k, b \ell}, A_{k, b \ell}, B_{k, b \ell},-F_{k, b \ell}, I\right)$ whose zeros are the poles of its inverse, that is, of the characteristic multipliers of the periodic pair $\left(A_{k, b \ell}+B_{k, b \ell} F_{k, b \ell}, E_{k, b \ell}\right)$ which all lie in the interior of the unit disk, because $F_{k, b \ell}$ is a stabilizing periodic feedback.

3) To show that $\mathcal{S}_{i, 1}$ is inner, we use the characterization of inner factors for standard periodic systems (i.e., with $E_{k}=$ $I$ ) in terms of the periodic observability Gramian [10]. For this we can show that for the periodic realization of $\mathcal{S}_{i, 1}=$ $\left(E_{k, i}, A_{k, i}, B_{k, i}, C_{k, i}, D_{k, i}\right)$ the following conditions of [10] are fulfilled:

$$
\begin{aligned}
A_{k, i}^{T} X_{k+1, b \ell} A_{k, i}+C_{k, i}^{T} C_{k, i} & =E_{k-1, i}^{T} X_{k, b \ell} E_{k-1, i} \\
B_{k, i}^{T} X_{k+1, b \ell} A_{k, i}+D_{k, i}^{T} C_{k, i} & =0 \\
D_{k, i}^{T} D_{k, i}+B_{k, i}^{T} X_{k+1, b \ell} B_{k, i} & =I
\end{aligned}
$$

\section{Conclusions}

The proposed computational method for determining inner-outer factorizations of causal periodic systems is completely general, being applicable to periodic systems with time-varying state dimensions, with arbitrary zeros and with a possibly non-injective input-output map (i.e., the corresponding lifted TFM has no full column rank). The proposed algorithm is lifting-free and operates exclusively on the matrices of the periodic state-space description. In a first step, structure preserving orthogonal similarity transformations are performed at the level of component matrices to determine a reduced system whose inner factor coincides with that of the original. For this system, a minimum phase factorization problem is solved, by solving a periodic Riccati equation. Reliable numerical algorithms for this purpose are described in [11]. Overall, the proposed method is numerically reliable, relying only on numerically stable or numerically reliable computational steps.

\section{APPENDIX}

\section{A. Periodic Kronecker-like form}

The system pencil $P_{k}^{\mathcal{S}}(z)$ of the lifted system (3) contains all information regarding system zeros and the Kroneckerstructure of the lifted TFM $G_{k}^{\mathcal{S}}(z)$. The same information is however provided by the block-permuted pencil

$$
\bar{P}_{k}^{\left(S_{k}, T_{k}\right)}(z):=\Pi_{1} P_{k}^{\mathcal{S}}(z) \Pi_{2}
$$

where $\Pi_{1}$ and $\Pi_{2}$ are suitable (orthogonal) block permutation matrices and

$$
\bar{P}_{k}^{\left(S_{k}, T_{k}\right)}(z):=\left[\begin{array}{ccccc}
S_{k} & -T_{k} & O & \cdots & O \\
O & \ddots & \ddots & \ddots & \vdots \\
\vdots & \ddots & \ddots & -T_{k+N-3} & O \\
O & & \ddots & S_{k+N-2} & -T_{k+N-2} \\
-z T_{k+N-1} & O & \cdots & O & S_{k+N-1}
\end{array}\right]
$$

with $S_{k}$ and $T_{k}$ defined in (10).

An algorithm for the reduction of the periodic pair $\left(S_{k}, T_{k}\right)$ to a periodic Kronecker-like form $\left(\bar{S}_{k}, \bar{T}_{k}\right):=$ $\left(Q_{k} S_{k} Z_{k}, Q_{k} T_{k} Z_{k+1}\right)$ using orthogonal periodic matrices $Q_{k}$ and $Z_{k}$ was proposed in [13]. Using this algorithm in conjunction with eigenvalue reordering techniques [14], we can determine, for example, a decomposition of the form

$$
\begin{aligned}
\bar{S}_{k} & =\left[\begin{array}{cccccc}
B_{k, r} & A_{k, r} & * & * & * & * \\
O & O & A_{k, g} & * & * & * \\
O & O & O & A_{k, b} & * & * \\
O & O & O & O & A_{k, \infty} & * \\
O & O & O & O & O & A_{k, \ell} \\
O & O & O & O & O & C_{k, \ell}
\end{array}\right], \\
\bar{T}_{k}= & {\left[\begin{array}{cccccc}
O & E_{k, r} & * & * & * & * \\
O & O & E_{k, g} & * & * & * \\
O & O & O & E_{k, b} & * & * \\
O & O & O & O & E_{k, \infty} & * \\
O & O & O & O & O & E_{k, \ell} \\
O & O & O & O & O & O
\end{array}\right], }
\end{aligned}
$$


where:

(a) $E_{k, r}$ is invertible and the periodic pair $\left(E_{k, r}^{-1} A_{k, r}, E_{k, r}^{-1} B_{k, r}\right)$ is completely reachable;

(b) $E_{k, \ell}$ is invertible and the periodic pair $\left(C_{k, \ell} E_{k-1, \ell}^{-1}, A_{k, \ell} E_{k-1, \ell}^{-1}\right)$ is completely observable;

(c) $A_{k, \infty}$ is invertible and the product $A_{k, \infty}^{-1} E_{k, \infty} \ldots A_{k+N-1, \infty}^{-1} E_{k+N-1, \infty}$ is nilpotent;

(d) $E_{k, g}$ and $E_{k, b}$ are non-singular, $\Psi_{E_{k, g}^{-1} A_{k, g}}$ has eigenvalues only in $\overline{\mathbb{D}}$, and $\Psi_{E_{k, b}^{-1} A_{k, b}}$ has only finite eigenvalues in $\mathbb{D}_{c}$.

The resulting reduced pencil

$$
\operatorname{diag}\left(Q_{k}, \ldots, Q_{k+N-1}\right) \bar{P}_{k}^{\left(S_{k}, T_{k}\right)}(z) \operatorname{diag}\left(Z_{k}, \ldots, Z_{k+N-1}\right)
$$

has the same structure as $\bar{P}_{k}^{\left(S_{k}, T_{k}\right)}(z)$ and allows to isolate subpencils (using only block permutations) which characterize particular sets of zeros or the left/right Kronecker structures. For example, the subpencil $\bar{P}_{k}^{\left(A_{k, g}, E_{k, g}\right)}(z)$ has only zeros in $\overline{\mathbb{D}}$, the subpencil $\bar{P}_{k}^{\left(A_{k, b}, E_{k, b}\right)}(z)$ has only zeros in $\mathbb{D}_{c}$, while $\bar{P}_{k}^{\left(A_{k, \infty}, E_{k, \infty}\right)}(z)$ has only infinite zeros. Also, the subpencils corresponding to the pairs $\left(\left[B_{k, r}, A_{k, r}\right],\left[E_{k, r}, 0\right]\right)$ and $\left(\left[\begin{array}{c}A_{k, \ell} \\ C_{k, \ell}\end{array}\right],\left[\begin{array}{c}E_{k, \ell} \\ 0\end{array}\right]\right)$ have the same right and left Kronecker structures as $\bar{P}_{k}^{\left(S_{k}, T_{k}\right)}(z)$, respectively.

\section{B. Computation of a special condensed form}

We use a reduction technique inspired from [1], which is adapted to the periodic matrix pairs $\left(S_{k}, T_{k}\right)$ in (10) with $E_{k}$ square and nonsingular.

First, we choose a periodic orthogonal matrix $Z_{k}^{(1)}$ such that

$$
\left[\begin{array}{ll}
C_{k} & D_{k}
\end{array}\right] Z_{k}^{(1)}=\left[\begin{array}{ll}
0 & \bar{D}_{k}
\end{array}\right]
$$

where $\bar{D}_{k}$ has full column rank $r_{k}$. We define

$$
\begin{aligned}
& S_{k}^{(1)}:=S_{k} Z_{k}^{(1)}=\left[\begin{array}{cc}
A_{k, 1} & A_{k, 2} \\
0 & \bar{D}_{k}
\end{array}\right], \\
& T_{k}^{(1)}:=T_{k} Z_{k+1}^{(1)}=\left[\begin{array}{cc}
E_{k, 1} & E_{k, 2} \\
0 & 0
\end{array}\right]
\end{aligned}
$$

where $\left[\begin{array}{ll}E_{k, 1} & E_{k, 2}\end{array}\right]$ has full row rank $n_{k+1}$.

We compute combining the algorithms proposed in [13] and [14] periodic orthogonal matrices $U_{k}$ and $Z_{k}^{(2)}$ to reduce the periodic pair $\left(A_{k, 1}, E_{k, 1}\right)$ to a periodic Kronecker-like form $\left(\widetilde{A}_{k, 1}, \widetilde{E}_{k, 1}\right)$ similar to (14) with

$$
\begin{aligned}
& \widetilde{A}_{k, 1}:=U_{k} A_{k, 1} Z_{k}^{(2)}=\left[\begin{array}{cc}
A_{k, r g} & { }^{*} \\
0 & \bar{A}_{k, b \ell}
\end{array}\right], \\
& \widetilde{E}_{k, 1}:=U_{k} E_{k, 1} Z_{k+1}^{(2)}=\left[\begin{array}{cc}
E_{k, r g} & * \\
0 & \bar{E}_{k, b \ell}
\end{array}\right],
\end{aligned}
$$

where the periodic pair $\left(A_{k, r g}, E_{k, r g}\right)$ contains the right Kronecker structure information and the zeros in $\overline{\mathbb{D}}$ (good zeros), while the periodic pair $\left(\bar{A}_{k, b \ell}, \bar{E}_{k, b \ell}\right)$ contains the left Kronecker structure information and the zeros in $\mathbb{D}_{c}$ (bad zeros).
Overall we have with $Q_{k}:=\operatorname{diag}\left(U_{k}, I_{p}\right)$

$$
\begin{aligned}
S_{k}^{(2)} & :=Q_{k} S_{k}^{(1)} \operatorname{diag}\left(Z_{k}^{(2)}, I_{r_{k}}\right)=\left[\begin{array}{ccc}
A_{k, r g} & { }^{*} & { }^{*} \\
0 & \bar{A}_{k, b \ell} & \bar{B}_{k, b \ell} \\
0 & 0 & \bar{D}_{k}
\end{array}\right] \\
T_{k}^{(2)}:=Q_{k} T_{k}^{(1)} \operatorname{diag}\left(Z_{k+1}^{(2)}, I_{r_{k}}\right) & =\left[\begin{array}{ccc}
E_{k, r g} & { }^{*} & { }^{*} \\
0 & \bar{E}_{k, b \ell} & \bar{F}_{k, b \ell} \\
0 & 0 & 0
\end{array}\right]
\end{aligned}
$$

where $\left[\bar{E}_{k, b \ell} \bar{F}_{k, b \ell}\right]$ has full row rank.

Finally, we compute orthogonal matrices $Z_{k}^{(3)}$ such that

$$
\left[\begin{array}{ll}
\bar{E}_{k, b \ell} & \bar{F}_{k, b \ell}
\end{array}\right] Z_{k+1}^{(3)}=\left[\begin{array}{ll}
E_{k, \ell} & 0
\end{array}\right]
$$

with $E_{k, \ell}$ invertible and define

$$
\begin{gathered}
\bar{S}_{k}:=S_{k}^{(2)} \operatorname{diag}\left(I, Z_{k}^{(3)}\right)=\left[\begin{array}{ccc}
A_{k, r g} & * & * \\
0 & A_{k, b \ell} & B_{k, b \ell} \\
0 & C_{k, b \ell} & D_{k, b \ell}
\end{array}\right] \\
\bar{T}_{k}:=T_{k}^{(2)} \operatorname{diag}\left(I, Z_{k+1}^{(3)}\right)=\left[\begin{array}{ccc}
E_{k, r g} & * & * \\
0 & E_{k, b \ell} & 0 \\
0 & 0 & 0
\end{array}\right]
\end{gathered}
$$

where $\left(\bar{S}_{k}, \bar{T}_{k}\right)=\left(Q_{k} S_{k} Z_{k}, Q_{k} T_{k} Z_{k+1}\right)$ with

$$
Z_{k}=Z_{k}^{(1)} \operatorname{diag}\left(Z_{k}^{(2)}, I_{r_{k}}\right) \operatorname{diag}\left(I, Z_{k}^{(3)}\right)
$$

The periodic system $\left(E_{k, b \ell}, A_{k, b \ell}, B_{k, b \ell}, C_{k, b \ell}, D_{k, b \ell}\right)$ with $p$ outputs and possibly time-varying number of inputs, has only zeros in $\mathbb{D}_{c}$ and the associated lifted system pencil has no right Kronecker structure.

\section{REFERENCES}

[1] C. Oară. Constructive solutions to spectral and inner-outer factorizations with respect to the disk. Automatica, 41:1855 - 1866, 2005.

[2] S. Bittanti and P. Colaneri. Periodic Systems: Filtering and Control. Springer-Verlag, Berlin, 2009.

[3] A. Varga. Computation of minimal periodic realizations of transferfunction matrices. IEEE Trans. Automat. Control, 46:146-149, 2004

[4] V.D. Estruch, V. Hernandez, E. Sanchez, and C. Coll. Forward/backward periodic realizations of nonproper rational matrices. J. Math. Syst. Estim. Control, 8:155175, 1998.

[5] A. Varga and P. Van Dooren. Computing the zeros of periodic descriptor systems. Systems \& Control Lett., 50:371-381, 2003.

[6] A. Varga. Strongly stable algorithm for computing periodic system zeros. In Proc. of CDC'2003, Maui, Hawaii, 2003.

[7] A. Varga. Computation of generalized inverses of periodic systems. In Proc. of CDC'04, Paradise Island, Bahamas, 2004.

[8] A. Varga. Computation of $\mathcal{L}_{\infty}$-norm of linear discrete-time periodic systems. In Proc. of MTNS'06, Kyoto, Japan, 2006.

[9] O. M. Grasselli and S. Longhi. Finite zero structure of linear periodic discrete-time systems. Int. J. Systems Sci., 22:1785-1806, 1991.

[10] B. Xie, R. K. A. V. Aripirala, and V. Syrmos. Model reduction of linear discrete-time periodic systems using hankel-norm approximations. In Proc. 13th IFAC Congress, San Francisco, USA, pages 245-250, 1996.

[11] A. Varga. On solving periodic Riccati equations. Numerical Linear Algebra with Applications, 15:809-835, 2008.

[12] K. Zhou, J. C. Doyle, and K. Glover. Robust and Optimal Control. Prentice Hall, 1996.

[13] A. Varga. Computation of Kronecker-like forms of periodic matrix pairs. In Proc. of MTNS'04, Leuven, Belgium, 2004.

[14] A. W. Bojanczyk, G. Golub, and P. Van Dooren. The periodic Schur decomposition. Algorithms and applications. In F. T. Luk, editor, Proceedings SPIE Conference, San Diego, volume 1770 of Advanced Signal Processing Algorithms, Architectures, and Implementations III, pages 31-42, July 1992. 